\title{
Entangled quantum histories
}

\section{Two chronicles of quantum mechanics tell a good tale but don't reflect the conflicts between the physicists who struggled to reconcile theory and fact, explains Don Howard.}

The nearly simultaneous publication of two popular histories of quantum mechanics, each highly readable and basically reliable, speaks well about the growing public interest in modern physics and about the commitment of major publishers to respond.

Louisa Gilder and Manjit Kumar take different approaches and emphasize different stages in the fascinating history of quantum physics. Kumar writes a conventional narrative history, focusing on the long-running debate between Niels Bohr and Albert Einstein, which took place from the mid-1920s through to the mid-1950s, over the adequacy of the quantum theory as a framework for fundamental physics. Gilder writes a delightfully unconventional history in the form of conversations - real or reconstructed - among the physicists themselves. She emphasizes the recent history of Bell's theorem, which concerns correlations between the quantum properties of separated elementary particles, its experimental tests and the subsequent exploitation of quantum entanglement in quantum computing, quantum information theory and quantum teleportation.

Gilder's is, on balance, the better book, partly because of the conversational format, which brings the scientist actors to life as complex personalities with interesting lives. Especially enjoyable are the portraits of the less famous physicists

\section{The Age of Entanglement: When Quantum Physics Was Reborn by Louisa Gilder Knopf: 2008. 464 pp. $\$ 27.50$}

Quantum: Einstein, Bohr, and the Great Debate about the Nature of Reality by Manjit Kumar Icon Books: 2008.480 pp. $£ 20$
Helmut Rechenberg - but he does not recognize where those histories have been questioned or eclipsed by more recent work. Gilder, by contrast, has read the newer specialist literature. She has also done her own research, such as tracking down an old physics colleague of Boris Podolsky, and eliciting from him the striking memory that Podolsky said he and Nathan Rosen never asked Einstein for permission before putting his name on the classic 1935 Einstein, Podolsky and Rosen paper, 'Can Quantum Mechanical Description of Physical Reality Be Considered Complete?'.

One must, however, be cautious about reconstructing conversations. Even the participants' own memories are not always to be trusted, the conversations recalled in Werner Heisenberg's Physics and Beyond (Harper and Row; 1971) being a notorious case in point. And when an author gathers quotations from letters, memoirs or published papers covering a modest span of years and reassembles them as one exchange, there is as much fiction as fact in the product. Gilder's imagined contents of the lively chat between Bohr and Einstein that, time and again, kept them missing their tram stop during Einstein's Copenhagen visit in 1923 is wonderful as who, starting in the 1960s, put entanglement to the test and taught us how to engineer with it, starting with John Bell and including Abner Shimony, John Clauser, Alain Aspect and Anton Zeilinger.

Gilder has done her homework. Kumar relies uncritically on an old generation of specialist histories - such as Max Jammer's classic The Conceptual Development of Quantum Mechanics (McGraw-Hill; 1966), or the multivolume history of quantum physics (Springer; 1982-1987) by Jagdish Mehra and stagecraft. Yet I ask myself how soon I'll see a student presenting it in a term paper as if it were a verbatim transcript.

Two larger reservations must be recorded about both books. The first concerns their treatment of Bohr and what is commonly termed the Copenhagen interpretation of quantum mechanics. Both Kumar and Gilder take as fact much of the folklore and mythology surrounding the Copenhagen interpretation. Legend has it that, from 1927 onwards, the entire Copenhagen community spoke with
Critical of quantum theory, Einstein (right) forced Bohr (left) and others to refine their thinking.

one voice about questions of interpretation. In fact, from the beginning, Bohr and Heisenberg disagreed about many fundamental points. Bohr never endorsed such famous bits of alleged Copenhagen orthodoxy as wavepacket collapse, a privileged role for the subjective observer, or a distinction between quantum and classical levels of description based solely on a system's scale. Heisenberg, who invented the term Copenhagen interpretation much later in 1955, asserted all of these claims. Bohr did not. Differences between Heisenberg and Bohr go almost unnoticed by both Kumar and Gilder, with unfortunate consequences for such crucial issues as understanding what really was at stake in the Bohr-Einstein debate and the consequences for Bohr of experimental tests of Bell's theorem. These tests provide strong evidence for quantum entanglement - the entwined states of interacting quantum systems - that was the basis of Bohr's own interpretation of quantum mechanics.

The second reservation concerns the consequences of experimental tests of Bell's theorem for another major interpretive project - David Bohm's construction in 1952 of a 'non-local hidden variables theory'. 
The theory posited unknown and perhaps inaccessible degrees of freedom of quantum systems, the values of which fix the behaviour of such systems. But quantum indeterminism is overcome at the price of allowing 'non-local' or 'faster-than-light' communication among those hidden variables, which might conflict with Einstein's theory of relativity. Gilder devotes long discussions to the career and ideas of Bohm. All the more puzzling, then, that when she tells us again and again about experiments confirming violations of the Bell inequality, she leaves the reader with the impression that this is a handsdown victory for quantum mechanics over hidden variable theories, when it is only local hidden variable theories that are put in jeopardy. One might reject Bohmian mechanics for other reasons, but these tests alone do not afford them. Kumar's hasty resumé of the same results leaves one still more confused. He suggests that Einstein's reservations about the completeness of quantum mechanics are somehow vindicated by experiments confirming the predictions of standard quantum mechanics.

Both authors would have been helped by a closer look at the well-known re-analysis of Bell's argument, introduced in 1984 by Jon Jarrett and refined by Shimony. Jarrett showed that the original Bell locality condition is a conjunction of two logically independent conditions that Shimony called outcome independence and parameter independence. The former is akin to a denial of quantum entanglement, the latter to relativistic locality constraints. Experimental violations of the Bell inequality can now be traced to violations of one or the other of these two conditions. That there are two independent routes to violations of the Bell inequality makes it clear how orthodox quantum mechanics, which presumes entanglement, and a Bohm-type hidden variables theory, which assumes relativistic non-locality at the microlevel, can both claim to have been vindicated by the Bell experiments. But these gentle criticisms should not deter the interested reader from enjoying two welcome additions to the popular history of twentieth-century physics.

Don Howard is professor of philosophy at the University of Notre Dame, Notre Dame, Indiana 46556, USA. His book on Einstein for Blackwell's Great Minds series will be published in 2009. e-mail:dhoward1@nd.edu

\section{Back to the roots of crop farming}

\section{Where Our Food Comes From: Retracing Nikolay Vavilov's Quest to End Famine by Gary Paul Nabhan \\ Island Press: 2008.266 pp. \$24.95, £21.50}

In 1941, when German and Finnish troops threatened to besiege the Russian city of Leningrad (now St Petersburg), Soviet leaders hurried to authorize the evacuation of the art collection from the city's Hermitage museum. Another extraordinary treasure, then the world's largest collection of more than 380,000 food crop samples housed at Leningrad's All-Union Institute of Agricultural Sciences, did not receive such privileged treatment; it survived the 1941-44 Leningrad blockade only through the virtue of committed individuals. Clearly, society places different values on the heritage content of the art museum and the seed bank.

In Where Our Food Comes From, Gary Paul Nabhan, a conservationist and research social scientist at the University of Arizona, spotlights crop diversity as a neglected but vital cultural resource. He does so by chronicling the journeys of Nikolay Vavilov, Russia's famous geneticist and botanist, and creator of the Leningrad seed bank. Vavilov was the first to identify the world's centres of crop diversity - a concept that, decades later, was developed by conservation biologists into what we now call biodiversity hotspots or conservation targets. Based on narratives from Vavilov's expeditions in the 1920s and 1930s and the author's own travels to retrace Vavilov's steps, Nabhan looks at changes in agricultural biodiversity.

The journeys cover nine regions of the globe. Each chapter highlights a specific land-use system, such as the wild apple forests of Kazakhstan; the diverse date cultivation in the oases of the Maghreb in north Africa; the traditional milpa cropping system in Mexico, based on maize (corn) and beans; or the forest gardens of

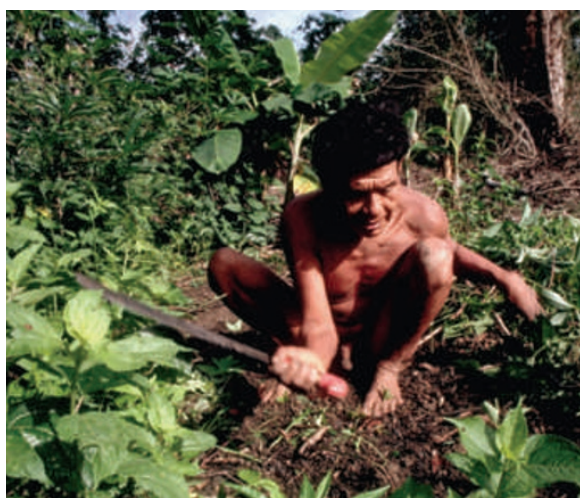

Amazon agriculture benefits from crop diversity. the Amazon basin in South America. Nabhan observes that, almost everywhere, crop diversity has strongly and rapidly declined during the time between Vavilov's and his own visits. Both global- and regional-scale processes are held responsible for the homogenization of crops. For example, melting glaciers account for a depletion of hydrological sources in Tajikistan's Pamir mountains, which damages traditional cropping systems. In northern Italy's Po valley, wasteful irrigation practices and mechanized high-performance agriculture have displaced traditional land uses. In the Middle East, political unrest and the legacy of colonialism have disrupted ancient farming systems. In Kazakhstan, economic growth has driven urban sprawl, threatening agricultural land close to cities.

Yet Nabhan's perspective is positive. He stresses the value of diverse crop varieties and the cultural traditions of land use and food preparation - for pragmatic reasons and as a cultural heritage that provides humankind with pleasure. He presents promising initiatives to recover crop diversity, from the rehabilitation of farmers' markets in Lebanon to efforts to renew local Hopi food systems in Arizona. And he shows how rich crop diversity and traditional seed selection and distribution support adaptation to changing environmental or societal conditions. I was fascinated by the story of how Colombian peasants have selected varieties of coca plants - the leaves of which contain cocaine alkaloids - that are resistant to the aerially sprayed herbicide used in the war on drugs. These resistant varieties have arisen through farmer-based selection, adaptation and informal dissemination of coca cuttings, all without any genetic engineering.

Where Our Food Comes From is a marked critique of the worldwide simplification of agricultural systems. It pins its hopes on local, traditional agriculture and is sceptical of topdown approaches to increasing food production, such as calls for another 'green revolution'. Many of its conclusions coincide with those reached by the International Assessment of Agricultural Knowledge, Science and Technology for Development, which concluded that the focus on maximum agricultural commodity production was responsible for the depletion of natural capital.

There are two opposing sides in the debate on how to increase food security. One side, which includes Nabhan, endorses the support of small-scale, low-output agriculture that is highly diverse. Among other examples, Nabhan uses the case of Ethiopian wheat varieties, which proved invaluable in the fight against 